\title{
Dietary fibre and the occurrence of gut symptoms in cystic fibrosis
}

\author{
J Gavin, J Ellis, A L Dewar, C J Rolles, G J Connett
}

\begin{abstract}
Objective-To evaluate the effect of currently recommended energy rich cystic fibrosis diets on fibre intake and to investigate the relationship between fibre intake and the occurrence of gut symptoms. Method-Prospective completion of nonweighed five day food diaries by 28 children with cystic fibrosis and comparison of mean daily fibre intake with age matched controls who did not have cystic fibrosis. Prospective completion of similar diaries to a total of 68 children with cystic fibrosis and comparison of fibre and lipase intake with the occurrence of gut symptoms.

Results-Mean daily fibre intake in children with cystic fibrosis was $7.00 \mathrm{~g}$ compared with $14.65 \mathrm{~g}$ in controls $(p<0.001)$. Mean daily fibre intake in eight patients troubled with moderate or severe abdominal pain was $0.144 \mathrm{~g} / \mathrm{kg}$. This was significantly lower $(\mathrm{p}<0.01)$ than mean values for 22 patients with occasional but mild symptoms $(0.249 \mathrm{~g} / \mathrm{kg})$ and 38 with no gut symptoms $(0.312 \mathrm{~g} / \mathrm{kg})$. There was a trend towards higher pancreatic enzyme doses (lipase/kg/day) in children with abdominal pain.

Conclusions-Currently recommended cystic fibrosis diets have a low fibre content. A low residue diet might be an important factor in the pathogenesis of gastrointestinal symptoms.

(Arch Dis Child 1997;76:35-37)
\end{abstract}

Keywords: cystic fibrosis; fibre; constipation.

Published recommendations for the nutritional management of children with cystic fibrosis are to encourage an energy dense diet to counteract energy losses from malabsorption and increased energy expenditure. ${ }^{1}$ Pancreatic enzymes are given to those with pancreatic insufficiency. Ideally dose requirements should be assessed by measurement of faecal fat or energy losses, but it is common practice to adjust the amounts given according to symptoms that suggest malabsorption, such as the frequent passage of abnormal stools and the occurrence of abdominal pain and distension. Until recently most physicians had a liberal approach to enzyme usage but this has been tempered by the occurrence of fibrosing colonopathy and its association with high doses of pancreatin. ${ }^{2}$

An alternative cause of abdominal pain in cystic fibrosis is extensive loading of the colon with faeces. This is commonly demonstrated on plain abdominal radiographs in symptomatic patients. ${ }^{3}$ Although this is a plausible cause of symptoms it is probably underrecognised and no explanation has been offered as to why this should occur so frequently in those with cystic fibrosis. In this study we set out to discover whether currently recommended cystic fibrosis diets lead to a reduction in fibre intake, an important factor in the aetiology of constipation, and to what extent fibre intake is associated with the occurrence of abdominal symptoms in patients with cystic fibrosis.

\section{Patients and methods}

STUDY 1

Twenty eight consecutive children (15 boys) attending annual assessments at our regional cystic fibrosis clinic were studied. The age range was 5-14 years. All had been regularly followed up by a cystic fibrosis dietitian and advised to eat an energy dense diet. Dietary fibre intake was estimated from food diaries kept over a five day period. Portion sizes were either accurately described using household measures or estimated using Food Portion Sizes. ${ }^{4}$ Data were analysed using the nutritional program COMP-EAT 4 (Nutrition Systems Ltd, London) to calculate a mean daily fibre intake of total non-starch polysaccharide (NSP) in $g$ for each patient.

Similar diary data were obtained from 28 age matched controls. These were recruited from healthy schoolchildren who had never received specific dietary advice or been referred for eating difficulties or poor weight gain. A two sample $t$ test was used to determine whether there were differences in fibre intake between the two study groups after the data had been tested for a normal distribution.

\section{STUDY 2}

Further diary data were obtained in 68 children (30 boys) by the methods in study 1 . The age range was $5-16$ years. Lipase intake was accurately recorded by the dietitian and the amounts taken calculated in terms of lipase/kg/day. Each child was independently assessed at the same clinic visit by the same paediatrician who recorded the presence of abdominal distension and the frequency and consistency of stools. The children were divided into three groups according to the occurrence of abdominal pain: group 1, no pain; group 2, occasional or mild discomfort; group 3, frequent and/or severe pain. Two sample $t$ tests were used to determine whether there were differences between fibre intake, 


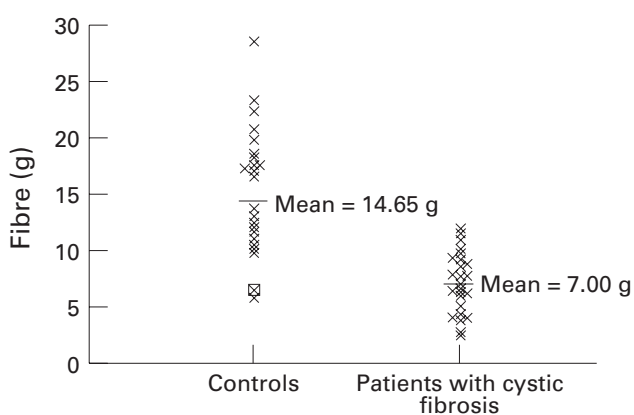

Figure 1 Daily dietary fibre intake of patients with cystic fibrosis and controls.

lipase intake, and the occurrence of gut symptoms between the three groups after testing the data for a normal distribution. Analyses were carried out using Minitab statistical software.

\section{Results}

STUDY 1

Mean (SD) daily fibre intake in children with cystic fibrosis was significantly lower at 7.00 (5.18) g compared with 14.65 (2.59) g in controls $(\mathrm{p}<0.0001)$ (fig 1 ).

STUDY 2

The mean daily fibre intake in the 68 children was $7.32 \mathrm{~g}$ (range 1.2-15.8, SD 3.48). Abdominal distension occurred in five children

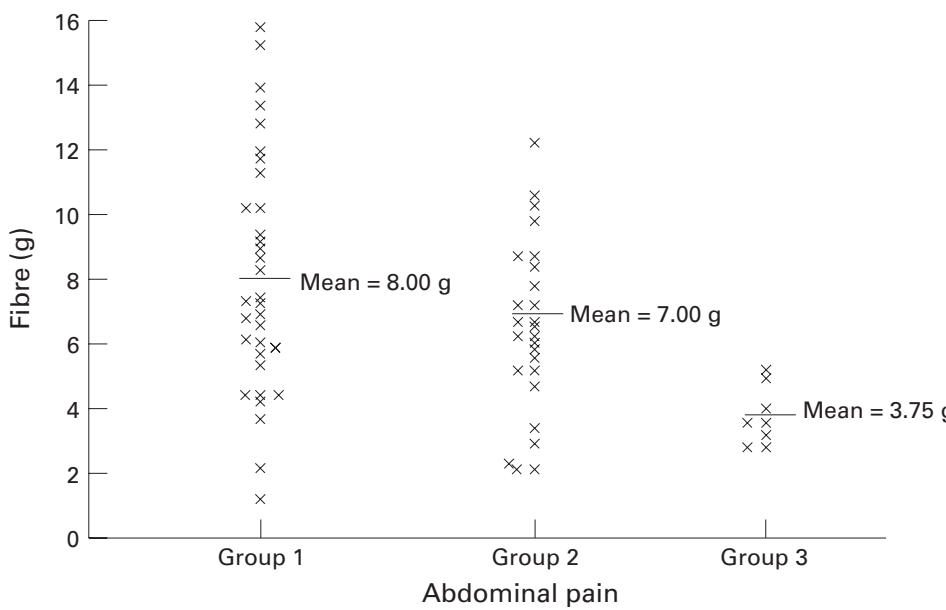

Figure 2 Dietary fibre intake plotted against occurrence of abdominal pain in patients with cystic fibrosis.

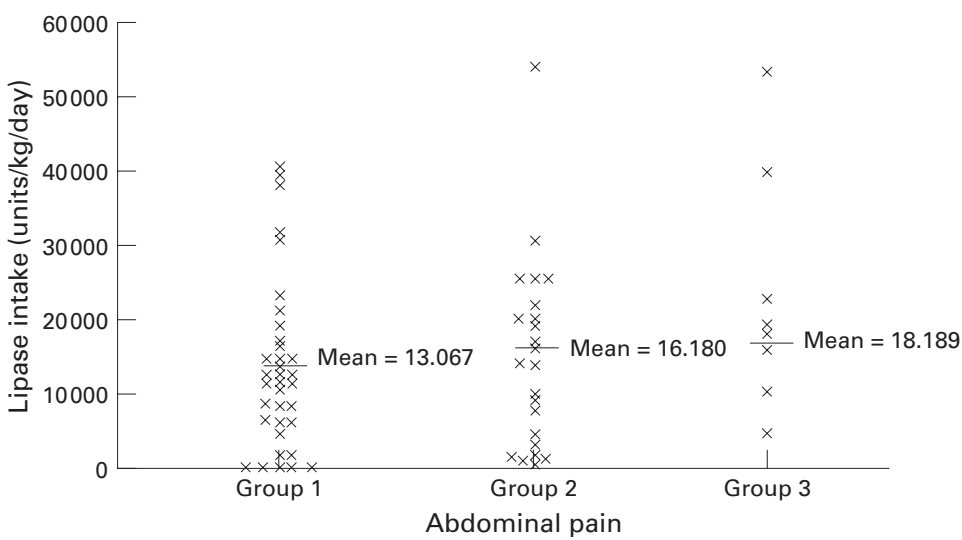

Figure 3 Lipase units/kg body weight/day plotted against occurrence of abdominal pain in patients with cystic fibrosis. and was not related to fibre or lipase intake. Similarly, there were no relationships between stool frequency or consistency and either of these two factors. When fibre intake was expressed in $\mathrm{g} / \mathrm{kg}$ body weight, those children with frequent and/or severe pain (group 3, $\mathrm{n}=8$ ) had a mean fibre intake of $0.144 \mathrm{~g} / \mathrm{kg}$. This was significantly lower than the fibre intake of 38 children with no pain (group 1; $0.312 \mathrm{~g} / \mathrm{kg}$ ) and 22 children with occasional or mild symptoms (group 2; $0.249 \mathrm{~g} / \mathrm{kg})(\mathrm{p}<0.01)$ (fig 2). Symptoms occurred evenly across the age range and the mean weights and weight SD scores of the children within each group were similar (group 1, 27.8 and -0.43 ; group 2, 32.16 and -0.28 ; group 3, 27.1 and -0.40 ).

There was a trend suggesting the use of higher enzyme doses in children with more severe gut symptoms but a wide range of doses was used within each group and the between group differences were not significant $(p>0.05)$ (fig 3).

\section{Discussion}

Current dietary practice in cystic fibrosis is to advocate energy dense foods to maximise growth potential. Our data suggest that as a result of these recommendations the intake of bulky low energy fibre foods tends to be low. The method we used in this study was to ask families to complete five day non-weighed food diaries. Although this method might be less accurate than keeping diaries of accurately weighed food intakes, we took this approach to ensure a high level of compliance. The fibre intake in the healthy age matched controls appears to be comparable to that reported elsewhere. The average intake of NSPs in 'healthy' adults in the UK was reported to be 11-13 g/day. ${ }^{5}$ There are no recommendations on ideal fibre intake for children, although a survey of UK adolescents suggests that they consume between 12 and $24 \mathrm{~g}$ of fibre daily. ${ }^{6}$

The fibre intake calculated in our study is total NSPs using the Englyst method. ${ }^{7}$ This relates to insoluble and soluble NSPs and does not include the unavailable carbohydrates cellulose and lignin. Up until recently, NSPs have not been thought to be important in the nutritional management of cystic fibrosis, although their importance in relation to colonic function has been recognised for many years. ${ }^{8}$ The relevant physiological functions of NSPs include increasing stool bulk as a result of their water holding capacity and increased growth of faecal bacteria, increasing colonic transit time by stimulating gut peristalsis, and their use as a substrate for bacterial fermentation to produce essential short chain fatty acids for colonic nutrition. ${ }^{9}{ }^{10}$

One could speculate that low dietary intakes of NSPs in children with cystic fibrosis might significantly compromise colonic function causing stasis of substrate, constipation, and abdominal pain. Furthermore a malnourished colon, lacking in short chain fatty acid nutrients, might be more vulnerable to other insults and at increased risk for the development of complications such as distal intestinal 
obstruction syndrome and fibrosing colonopathy. ${ }^{11}$

It is common for physicians to attribute gut symptoms in patients with cystic fibrosis to pancreatic enzyme insufficiency. The possibility that a low residue diet might be an important causal factor has not been recognised until recently. Such symptoms might be improved by increasing fibre intake, but the risks of doing this would be to reduce energy intake and compromise overall nutrition. This approach would only be appropriate in children with normal growth and appetite and under strict dietetic supervision. Given such circumstances, high fibre foods could be usefully introduced as part of main meals with snacks remaining as high energy alternatives.

It is still not clear which of the individual constituents in fibre are of particular benefit, and some researchers have advised a cautious approach to the widespread promotion of fibre enhanced foods. ${ }^{12}$

One group of patients with cystic fibrosis not included in this study are those receiving enteral nutrition. This group are often anorexic, suffer gut symptoms, and invariably have a very low fibre intake. Such children might well benefit from the use of fibre supplemented enteral feeds without compromising oral energy intake.
Modern cystic fibrosis diets have been of undoubted benefit in maintaining optimal growth and nutritional status. Further research is needed to confirm that current practices do lead to a low fibre intake and to increase understanding about the clinical importance of a low fibre diet on colonic function.

1 MacDonald A. Nutritional management of cystic fibrosis. Arch Dis Child 1996;74:81-7.

2 Smyth RL. Fibrosing colonopathy in cystic fibrosis. Arch Dis Child 1996;74:464-8.

3 Littlewood JM. Gastrointestinal complications in cystic fibrosis. Br Med Bull 1992;48:847-59.

4 Ministry of Agriculture, Fisheries, and Food. Food portion sizes. London: HMSO, 1993.

5 Department of Health. Dietary reference values for food, energy and nutrients for the United Kingdom. Report No 41. London: HMSO, 1991.

6 Department of Health. The diets of British school children. Report No 36. London: HMSO, 1989.

7 Englyst HN, Cummings JH. An improved method for the Englyst $\mathrm{HN}$, Cummings $\mathrm{JH}$. An improved method for the
measurement of dietary fibre as the non-starch polysacchameasurement of dietary fibre as the non-starch polysaccha-
rides in plant foods. Fournal of the Association of Official rides in plant foods. Fournal of the
Agricultural Chemists 1988;71:808-14

Agricultural Chemists 1988;71:808-14.
8 Cummings JH, Englyst HN. Fermentation in the human large intestine and the available substrates. Am $\mathcal{F}$ Clin Nutr 1987;45:1243-55

9 Sunvold GD, Titgemeyer EC, Bourquin LD, Fahey GC, Garleb KA. Alteration of the fibre and lipid components of defined formula diet: effects on stool characteristics, nutrient digestibility, mineral balance, and energy metabolism in humans. Am f Clin Nutr 1995;62:1252-60.

10 Scheppach W, Burghardt W, Bartram P, Kasper H. Addition of dietary fibre to liquid formula diets: the pros and cons. fournal of Parenteral and Enteral Nutrition 1990;14:204-9.

11 Dodge JA. The aetiology of fibrosing colonopathy. Postgrad Med F 1996; 72(suppl 2):S52-5.

12 Wasan HS, Goodland RA. Fibre-supplemented foods may damage your health. Lancet 1996;348:319-20. 